\title{
Constraining the spin-down timescale of the white-dwarf progenitors of Type Ia supernovae
}

\author{
Xiangcun. Meng \\ School of Physics and Chemistry, Henan Polytechnic University, Jiaozuo, 454000, China \\ Key Laboratory for the Structure and Evolution of Celestial Objects, Chinese Academy of Sciences, \\ Kunming 650011, China \\ xiangcunmeng@hotmail.com \\ and \\ Philipp. Podsiadlowski \\ Department of Astronomy, Oxford University, Oxford OX1 3RH
}

\begin{abstract}
Justham (2011) and diStefano et al. (2011) proposed that the white-dwarf progenitor of a Type Ia supernova (SN Ia) may have to spin down before it can explode. As the white dwarf spin-down timescale is not well known theoretically, we here try to constrain it empirically (within the framework of this spin-down model) for progenitor systems that contain a giant donor and for which circumbinary material has been detected after the explosion: we obtain an upper limit of a few $10^{7} \mathrm{yr}$. Based on the study of Di Stefano \& Kilic (2012), this means that it is too early to rule out the existence of a surviving companion in SNR 0509-67.5.
\end{abstract}

Subject headings: binaries: symbiotic - stars: evolution- star: mass-loss - supernovae: general - star: white dwarfs

\section{INTRODUCTION}

Type Ia supernovae (SNe Ia) appear to be good cosmological distance indicators and have been successfully used for determining cosmological parameters (e.g. $\Omega$ and $\Lambda$ ); this has led to the discovery that the Universe is accelerating (Riess et al. 1998; Perlmutter et al. 1999). It has also been proposed that they can be used to test the evolution of the dark energy equation of state with time (Howell et al. 2009). However, the progenitor systems of SNe Ia have not yet been confidently identified (Hillebrandt \& Niemever 2000; Leibundgut 2000), although the identification of the progenitor would be important in many astrophysical fields (Wang \& Han 2012). Two basic scenarios have been competing for about four decades. In the single degenerate (SD) model, a carbonoxygen white dwarf (CO WD) grows in mass via accretion from its non-degenerate companion
(Whelan \& Iben 1973; Nomoto et al. 1984), while in the double degenerate (DD) scenario, two WDs merge after losing angular momentum by gravitational wave radiation (Iben \& Tutukov 1984; Webbink 1984).

Searching for the surviving companion in a supernova remnant (SNR), only predicted in the SD scenario, provides one of the most promising methods for distinguishing between the two basic scenarios. The claim of the discovery of a potential surviving SN companion in the Tycho supernova remnant has been hailed as a major advance in this field (Tycho G, Ruiz-Lapuente et al. 2004), although serious doubts have been raised that Tycho G actually is the surviving companion (Kerzendorf et al. 2009, 2013; González-Hernández et al. 2009; Shappee et al. 2013). Recently, Schaefer \& Pagnotta (2012) searched for a potential surviving companion in 
SNR 0509-67.5 and reported a negative result, apparently favoring the DD model. On the other hand, Di Stefano \& Kilic (2012) argued that, if the WD experienced a long spin-down phase before the explosion, the donor star could be too dim to be detectable at the time of explosion. Unfortunately, the spin-down timescale is very uncertain (Di Stefano et al. 2011), and it is therefore difficult to arrive at a firm conclusion with regard to the observations by Schaefer \& Pagnotta (2012).

There has been evidence for circumstellar material (CSM) in the spectrum of a number of SNe Ia, which is usually taken as evidence in favor of the SD model (Patat et al. 2007; Sternberg et al. 2011; Dilday et al. 2012; Maguire et al. 2013). The fact that the CSM can be detected indicates that the companions are still losing material before the SN Ia explosion or only ceased transferring mass relatively recently before the explosion. This implies that the spin-down timescale should be shorter than the timescale on which the companion loses all of its remaining envelope; otherwise the signature of the CSM should not be detected. In this paper, we want to use this idea to empirically constrain the spin-down timescale (within the framework of the spin-down model).

In Section 2, we describe our method and present the results of our calculations in Section 3. In Section 4, we discuss their implications.

\section{METHOD}

If the WD companion is a main-sequence (MS) star, the wind mass-loss rate will be relativley low and the velocity of outflow from the binary system very high; and it is then very difficult to detect the CSM. Therefore, we only consider WD + red giant (RG) systems here. Although the details of detecting the CSM from WD + RG systems depend on the system parameters, the CSM has be detected in at least some systems (Patat et al. 2007; Dildav et al. 2012). We will address this problem again in another future paper. The method to follow the binary evolution here is very similar to the prescriptions presented in Chen et al. (2011)

We use the stellar evolution code of Eggleton (1971) to calculate the binary evolutions of SD systems. Roche lobe overflow (RLOF) is treated within the code as described by Han et al. (2000). A 'standard' solar metallicity is adopted $(Z=$
0.02). The opacity tables for the metallicity come from the compilations from Chen \& Tout (2007), Iglesias \& Rogers (1996) and Alexander \& Ferguson (1994).

We also assume that the stellar wind mass-loss rate of the secondary in a binary system is increased by the presence of the WD companion star. Specifically, the tidal enhancement of massloss rate from secondary is modelled using the Reimers' (1975) wind-mass formula with an extra tidal term following Tout \& Eggleton (1988):

$$
\begin{aligned}
\dot{M}_{2 \mathrm{w}} & =-4 \times 10^{-13} \frac{\eta\left(L / L_{\odot}\right)\left(R / R_{\odot}\right)}{\left(M_{2} / M_{\odot}\right)} \\
& \times\left[1+B_{\mathrm{W}} \min \left(\frac{1}{2}, \frac{R}{R_{\mathrm{L}}}\right)^{6}\right] M_{\odot} \mathrm{yr}^{-1},
\end{aligned}
$$

where $L$ and $R$ are the luminosity and the radius of the giant secondary, $R_{\mathrm{L}}$ is its Roche lobe radius, and $\eta=0.25$ is the Reimers' wind coefficient. The wind enhance factor $B_{\mathrm{W}}$ is still uncertain; it is more than 3000 in Tout \& Eggleton (1988) and $10^{4}$ in the wind-driven mass transfer theory of Tout \& Hall (1991). Here, we set $B_{\mathrm{W}}=10000$, which means that the mass-loss rate from the secondary could be 150 times as large as the Reimers' rate when the star fills more than half its Roche lobe.

Some of the material lost in the stellar wind from the secondary may be accreted by the WD; the resulting mass-accretion rate is expressed as (from Boffin \& Jorissen 1988)

$\dot{M}_{2 \mathrm{a}}=-\frac{1}{\sqrt{1-e^{2}}}\left(\frac{G M_{\mathrm{WD}}}{v_{\mathrm{w}}^{2}}\right)^{2} \frac{\alpha_{\mathrm{acc}} \dot{M}_{2 \mathrm{w}}}{2 a^{2}\left(1+v_{\mathrm{orb}}^{2} / v_{\mathrm{w}}^{2}\right)^{3 / 2}}$,

where $v_{\text {orb }}=\sqrt{G\left(M_{2}+M_{\mathrm{WD}}\right) / a}$ is the orbital velocity, $G$ Newton's gravitational constant, $a$ the semi-major axis of the orbit, and $e$ its eccentricity. In this paper, we take $e=0$. The accretion efficiency $\left(\alpha_{\text {acc }}\right)$ is set as 1.5. For simplicity, we set $v_{\mathrm{w}}=500 \mathrm{~km} \mathrm{~s}^{-1}$ for a MS star and $5 \mathrm{~km} \mathrm{~s}^{-1}$ for a RG. Here, $5 \mathrm{~km} \mathrm{~s}^{-1}$ is a lower limit for the wind velocity (see also Chen et al. 2011). In this equation, for sufficiently small $a$, the right-hand side can be larger than $-\dot{M}_{2 \mathrm{w}}$; we therefore limit $\dot{M}_{2 \mathrm{a}} \leq-\dot{M}_{2 \mathrm{w}}$, as did Chen et al. (2011). In fact, both $B_{\mathrm{W}}$ and $v_{\mathrm{w}}$ are poorly known and only upper or lower limits are used here for $B_{\mathrm{W}}$ and $v_{\mathrm{w}}$, 
respectively. Chen et al. (2011) have shown that the parameter space leading to SNe Ia in the orbital period-secondary mass plane increases with $B_{\mathrm{W}}$ and decreases with $v_{\mathrm{w}}$. Since $B_{\mathrm{W}}=10000$ and $v_{\mathrm{w}}=5 \mathrm{~km} \mathrm{~s}^{-1}$ may be considered to be upper and lower limits for the two parameters, respectively, the resulting parameter space leading to SNe Ia in the orbital period-secondary mass plane is the most generous case and should cover those from other parameter combinations. We will discuss their effects on the results, as well as possible metallicity effects, in section 4.1.

Wind accretion is the only way to transfer material from the RG secondary to the WD before Roche lobe overflow (RLOF) begins; then the mass-transfer rate is $\dot{M}_{\mathrm{tr}}=\dot{M}_{2 \mathrm{a}}$. After RLOF has started, material is transferred by both an accretion stream from the inner Lagrangian point and the wind so that $\dot{M}_{\mathrm{tr}}=\dot{M}_{2 \mathrm{a}}+\left|\dot{M}_{2 \mathrm{RLOF}}\right|$, where $\dot{M}_{2 \mathrm{RLOF}}$ is the mass-transfer rate by RLOF.

We adopt the prescription of Hachisu et al. (1999a) on WDs accreting hydrogen-rich material from their companions (see details in Han \& Podsiadlowski 2004 and Meng et al. 2009). Then, the mass growth rate of the CO WD, $\dot{M}_{\mathrm{WD}}$, is

$$
\dot{M}_{\mathrm{WD}}=\eta_{\mathrm{He}} \eta_{\mathrm{H}} \dot{M}_{\mathrm{tr}}
$$

where $\eta_{\mathrm{H}}$ is the mass-accumulation efficiency for hydrogen burning and is controlled by

$$
\eta_{\mathrm{H}}= \begin{cases}\dot{M}_{\mathrm{c}} / \dot{M}_{\mathrm{tr}}, & \dot{M}_{\mathrm{tr}}>\dot{M}_{\mathrm{c}}, \\ 1, & \dot{M}_{\mathrm{c}} \geq \dot{M}_{\mathrm{tr}} \geq \frac{1}{8} \dot{M}_{\mathrm{c}} \\ 0, & \dot{M}_{\mathrm{tr}}<\frac{1}{8} \dot{M}_{\mathrm{c}}\end{cases}
$$

where $\dot{M}_{\mathrm{c}}$ is the critical accretion rate for stable hydrogen burning; $\eta_{\mathrm{He}}$ is the mass-accumulation efficiency for helium-shell flashes, and its value is taken from Kato \& Hachisu (2004). The material lost in the form of an optically thick wind (Hachisu et al. 1996) is assumed to take away the specific orbital angular momentum of the accreting WD, while the wind material from the secondary that is not accreted by the WD is assumed to take away the specific orbital angular momentum of the donor star. For simplicity, we choose $M_{\mathrm{WD}}^{\mathrm{i}}=1.0 M_{\odot}$ and $1.1 M_{\odot}$, while $M_{2}^{\mathrm{i}}$ ranges from $0.8 M_{\odot}$ to $5.6 M_{\odot}$ in steps of $0.2 M_{\odot}$ and the initial orbital period from $\log \left(P^{\mathrm{i}} /\right.$ day $)=1.5$ to 3.5 in steps of 0.1 . In the calculations, we assume that a WD may explode as a SN Ia when its mass exceeds $1.378 M_{\odot}$ (Nomoto et al. 1984). At the moment when $M_{\mathrm{WD}}=1.378 M_{\odot}$, the companions are ascending either the first-giant branch (FGB) or the asymptotic giant branch (AGB). We then record the status of the companions at this moment, i.e. the secondary mass $M_{2}^{\mathrm{SN}}$, the core mass $M_{\text {core }}$ and the mass-loss rate from the secondary $\dot{M}_{2}$. The definition of the core is the same as that in Han et al. (1994) and Meng et al. (2008). We then estimate the remaining mass-loss timescale of the secondary from

$$
t=\frac{M_{\mathrm{env}}}{\left|\dot{M}_{2}\right|}=\frac{M_{2}^{\mathrm{SN}}-M_{\mathrm{core}}}{\left|\dot{M}_{2}\right|} .
$$

The mass-loss timescale here should be taken as the upper limit for the spin-down timescale and we assume that it is the spin-down timescale. The real mass-loss timescale could be different from the value here since $\dot{M}_{2}$ is the value at the moment when $M_{\mathrm{WD}}=1.378 M_{\odot}$, but the results here should be reasonable, at least, to first order.

\section{RESULTS}

Figure 1 shows the core masses and the envelope masses of secondaries at the moment when $M_{\mathrm{WD}}=1.378 M_{\odot}$. The secondaries can be clearly divided into three groups: $M_{\text {core }}<0.46 M_{\odot}$, $0.5 M_{\odot}<M_{\text {core }}<0.6 M_{\odot}$ and $M_{\text {core }}>0.8 M_{\odot}$. For the first group, the initial secondary mass $M_{2}^{\mathrm{i}}$ is smaller than $2.0 M_{\odot}$ and the core is composed of helium, while $M_{2}^{\mathrm{i}}$ in the second group ranges from $2.0 M_{\odot}$ to $2.4 M_{\odot}$ and the core is made of CO. $M_{2}^{\mathrm{i}}$ in the third group is larger than $4.6 M_{\odot}$, up to $5.6 M_{\odot}$, and the core is also made of CO (see also Figure 2 in Chen et al. 2011). The third group is very parameter dependent and will disappear for lower values of $M_{\mathrm{WD}}^{\mathrm{i}}$ and $B_{\mathrm{W}}$ (Chen et al. 2011), which indicates again that the results here are conservative (i.e. include all possibilities). For those with $M_{2}^{\mathrm{i}} \leq 2.4 M_{\odot}$, the envelope mass at the moment of $M_{\mathrm{WD}}=1.378 M_{\odot}$ is usually lower than $1 M_{\odot}$, and may be as low as $0.01 M_{\odot}$, while for those with $M_{2}^{\mathrm{i}} \geq 4.6 M_{\odot}$, the envelope mass may be as high as $3.4 M_{\odot}$. If the secondary does not lose all of its envelope before the supernova explosion, the envelope may be stripped off by the supernova ejecta (Marietta et al. 2000; Meng et al. 2007). Therefore, after the interaction between the supernova ejecta and the secondary, the final 


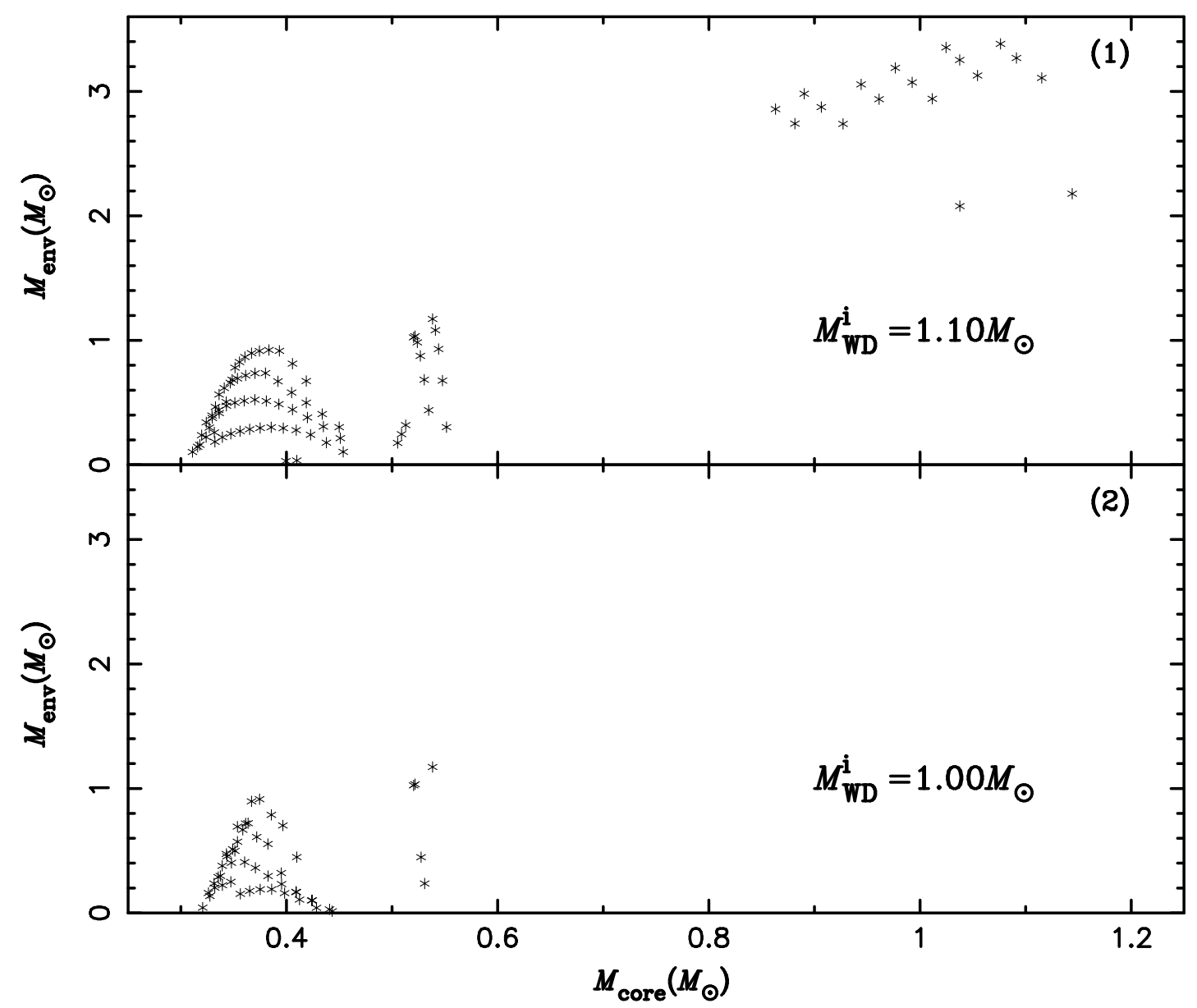

Fig. 1.- The core masses and the envelope masses of secondaries for different initial WD mass when $M_{\mathrm{WD}}=1.378 M_{\odot}$. 
remnant would be a helium or CO WD for the first and second groups, respectively. For the third group, there could be thin helium layer above the CO core (Meng et al. 2008), and the final remnant could then be a CO WD or a helium RG.

Figure 2 shows the mass-loss timescale as a function of mass-loss rate of the secondary at the moment when $M_{\mathrm{WD}}=1.378 M_{\odot}$. The mass-loss timescale here is still very uncertain although the uncertainty is much smaller than that quoted in Di Stefano \& Kilic (2012). Almost all systems have a mass-loss timescale shorter than $10^{7} \mathrm{yr}$ and some are even below $10^{5} \mathrm{yr}$. The mass-loss rate of the secondary $\left|\dot{M}_{2}\right|$ is always larger than $10^{-7} M_{\odot} \mathrm{yr}^{-1}$, even as high as $10^{-5} M_{\odot} \mathrm{yr}^{-1}$, which indicates that the mass loss from some binary systems at the moment when $M_{\mathrm{WD}}=1.378 M_{\odot}$ is dominated by the optically thick wind.

Figure 2 shows that the mass-loss timescale is much shorter than the evolutionary timescale of the secondary, (i.e. from the ZAMS to the moment of $\left.M_{\mathrm{WD}}=1.378 M_{\odot}\right)$. So, we may take the evolutionary timescale of the secondary as the delay time of the SNe Ia in the WD + RG channel. In Figure 3, we show the mass-loss timescale as a function of the evolutionary timescale of the secondary. The evolutionary timescale of the secondary covers a very large interval, i.e. from less than $10^{8} \mathrm{yr}$ to more than $10^{10} \mathrm{yr}$, which implies that the WD + RG channel may produce the youngest, middle age and the oldest SNe Ia as well. In addition, there is an interesting trend that the upper boundary of the mass-loss timescale seemingly decreases with the evolutionary timescale of the secondary; this is a consequence from the fact that the star with a larger initial mass tends to leave a more massive envelope when its WD companion has grown to $1.378 M_{\odot}$. This might indicate that it is more likely to detect the signal of the CSM in a younger population.

\section{DISCUSSION AND CONCLUSIONS}

\subsection{Uncertainties}

Yoon \& Langer (2004, 2005) found that if the accretion rate onto a CO WD is larger than $10^{-7}$ $M_{\odot} \mathrm{yr}^{-1}$, the WD may rotate strongly differentially (though this depends on exactly how angular momentum is being redistributed within the WD); this allows the WD to increase its mass up to $\sim 2$
$M_{\odot}$ before it can explode as a SN Ia or collapse to a neutron star by electron capture. Chen \& Li (2009) and Hachisu et al. (2012) puts these results into the canonical SD model to try explain the origin of overluminous SNe Ia (SN 2003fglike), often referred to as 'super-Chandra' $\mathrm{SNe}$ Ia. However, in the simulations of Yoon \& Langer (2004, 2005), the material that was accreted was carbon/oxygen-rich material. Can we directly apply these results to the case on accretion of hydrogen-rich material? If so, are the results the same for hydrogen or helium burning on a rotating WD and a non-rotating WD? Yoon et al. (2004) investigated the effects of rotation on the heliumburning shell in an accreting WD and found that helium burning in their rotating models is much more stable than in the non-rotating model because helium is ignited under much less degenerate conditions in the rotating models. Then, how can this be applied to hydrogen burning?

To explain the absence of hydrogen lines in the nebular spectrum of SNe Ia (Leonard 2007), Justham (2011) and Di Stefano et al. (2011) proposed that the CO WD may experience a long spin-down period to lose the angular momentum gained from the accreted material . This model may also explain the absence of detectable surviving companions in SN Ia remnants (Justham 2011; Di Stefano \& Kilic 2012). However, there is the more general question whether a WD can gain angular momentum by accretion to spin itself up significantly. Di Stefano \& Kilic (2012) pointed to a sample of fast spinning WDs in close binary systems, but mostly in so-called intermediate polars, which could mean that a strong magnetic field may be necessary to spin up the WD. However, it is still unclear whether a WD may effectively spin-up by gas friction without magnetic field.

Assuming that the WD can be spun up by the accretion of hydrogen-rich material and the hydrogen-rich material may be burned into helium and then carbon/xoygen to increase the WD mass, when does the WD begin to spin down? An accretion rate of $3 \times 10^{-7} M_{\odot} \mathrm{yr}^{-1}$ was used as the threshold in Chen \& Li (2009), while it is $1 \times 10^{-7}$ $M_{\odot} \mathrm{yr}^{-1}$ in Hachisu et al. (2012) and Justham (2011). However, these values are based on the accretion of carbon/oxygen-rich material from Yoon \& Langer (2004, 2005). It should be worth checking whether the results in Yoon \& Langer 


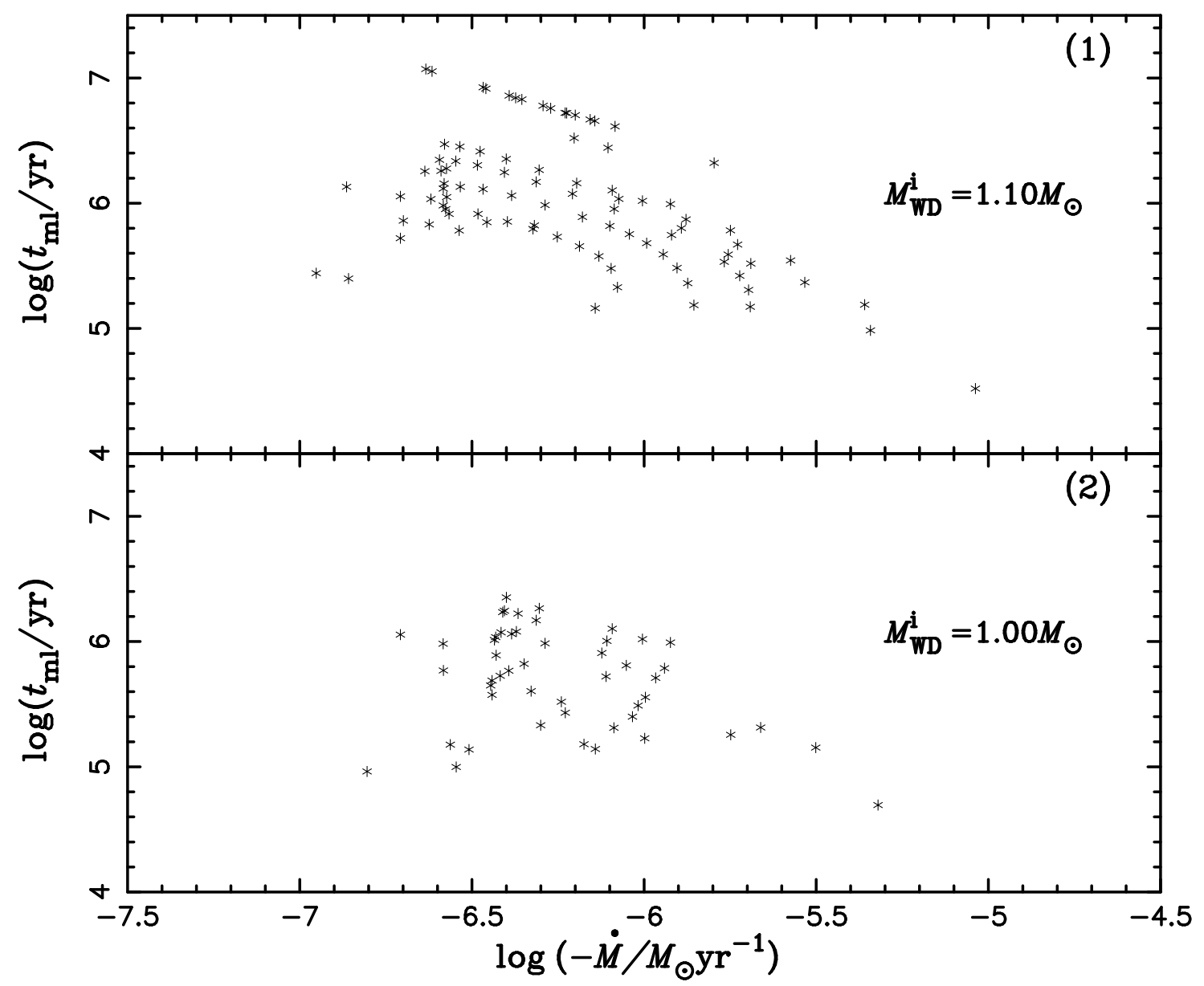

Fig. 2.- The mass-loss timecale as a function of mass-loss rate of the secondary at the moment when $M_{\mathrm{WD}}=1.378 M_{\odot}$. 


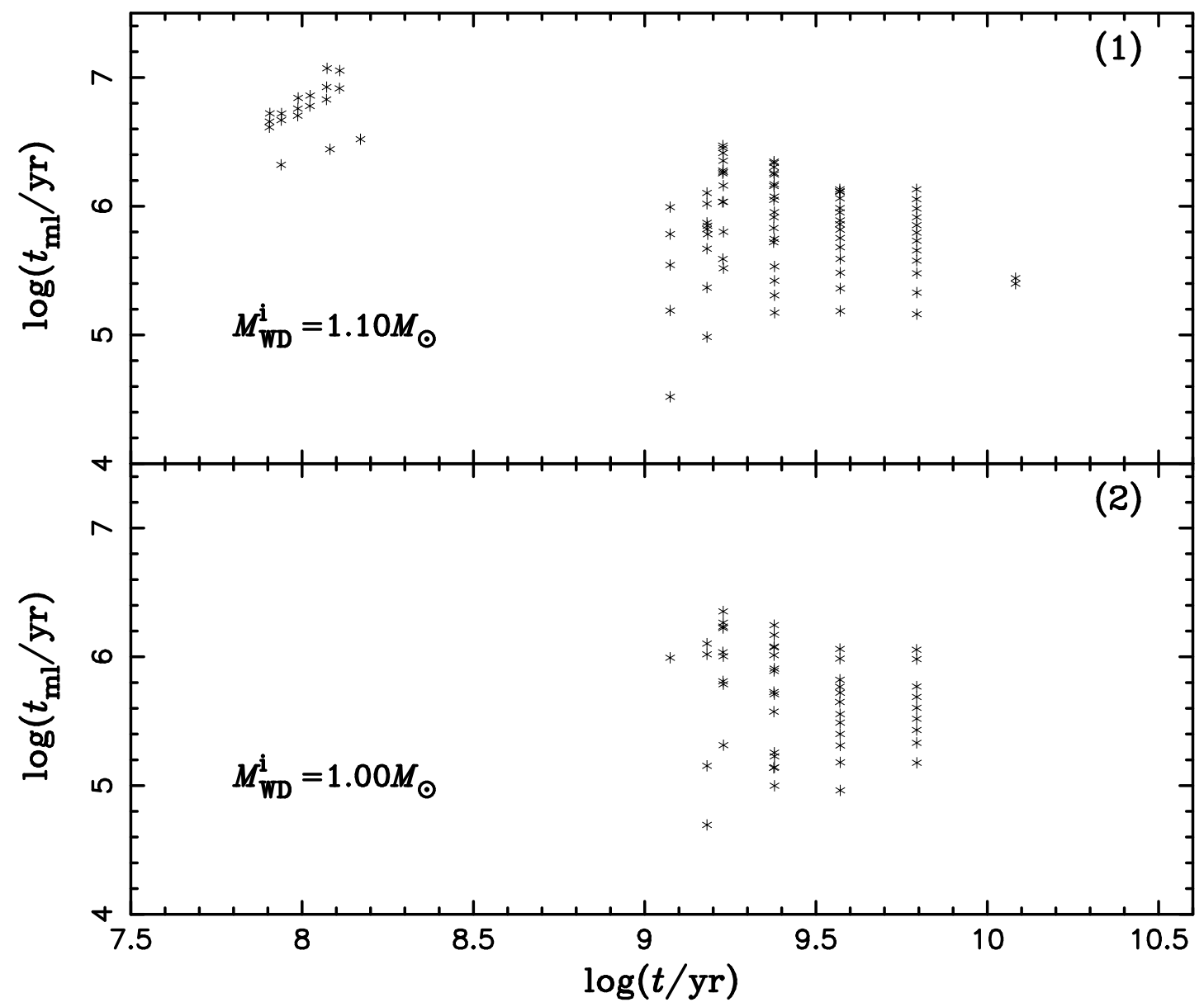

Fig. 3. - The mass-loss timescale as a function of the evolutionary timescale of the secondary at the moment when $M_{\mathrm{WD}}=1.378 M_{\odot}$. 
(2004, 2005) can be generalized to the case of the accretion of hydrogen-rich material, i.e. when does the WD begin to spin down for the accretion of hydrogen-rich material? In this paper, we just use $M_{\text {env }} /\left|\dot{M}_{2}\right|$ to constrain the spin-down timescale at the moment of $M_{\mathrm{WD}}=1.378 M_{\odot}$. If the WD begins to spin down before this moment, the WD could be rigidly rotating, and a very long spin-down timescale is expected $\left(\geq 10^{9} \mathrm{yr}\right.$, Hachisu et al. 2012). Then, the uncertainties in our method would be small and could be neglected $(0.1 \%)$. On the other hand, if the WD begins to spin down after $M_{\mathrm{WD}}=1.378 M_{\odot}$ has been reached, the time scale obtained here should be taken as an upper limit. In addition, since $\left|\dot{M}_{2}\right|$ usually increases when the companion star climbs the FGB/AGB, the $\left|\dot{M}_{2}\right|$ used here should generally be lower than the real value. So, the timescale obtained in this paper should be taken as an upper limit of the required spin-down timescale.

So far, we only used one particular set for the poorly determined paramters $\left(B_{\mathrm{W}}=10000\right.$ and $v_{\mathrm{W}}=5 \mathrm{~km} \mathrm{~s}^{-1}$ ) to model the problem. Chen et al. (2011) have shown that the parameter space leading to SNe Ia in the orbital period-secondary mass plane decreases with decreasing $B_{\mathrm{W}}$ or increasing $v_{\mathrm{W}}$. In Figure 4, we show the effect of varying these parameters, as well as metallicity, on the mass-loss timescale. This figure shows that the upper boundary of the mass-loss timescale is not significantly affected by $v_{\mathrm{W}}$ and metallicity, but the influence of $B_{\mathrm{W}}$ is large. Generally, a low $B_{\mathrm{W}}$ results in a lower wind mass-loss rate, which means that the companion in a given binary system has a thicker envelope when it fills its Roche lobe and it is more likely to experience dynamically unstable mass transfer. So, the upper boundary of the initial companion mass for SNe Ia moves to a lower value with decreasing $B_{\mathrm{W}}$, in particular, the third group in Fig. 1 disappears (also see Figure 3 in Chen et al. 2011) and, as a consequence, the companion has a less massive envelope when $M_{\mathrm{WD}}=1.378 M_{\odot}$, which results in a lower massloss timescale. Actually, lower values of $v_{\mathrm{W}}$ and $Z$ may also lead to a slightly lower upper boundary of the mass-loss timescale for a low initial WD mass, but the effect is not as significant as for $B_{\mathrm{W}}$. For the lower boundary of the mass-loss timescale, it is mainly determined by the minimum envelope mass required to maintain effective accretion onto the WD. In general, the mass of the minimum envelope mass is similar for all values of $B_{\mathrm{W}}, v_{\mathrm{W}}$ and $Z$.

We also note that we did not consider the dispersion time of the CSM $\left(10^{4} \sim 10^{5} \mathrm{yr}\right)$ and the simmering time $\left(10^{3}\right)$ in the core of the WD before the supernova explosion, since they can be neglected relative to the upper limit of the massloss timescale.

For the discussion above, $10^{7}$ yr should be a conservative upper limit of the spin-down timescale. On the theoretical side, the spin-down timescale is very uncertain; it is mainly determined by the timescale of the redistribution or loss of angular momentum in the WD, whether it is rotating differentially or as a solid body. The timescale for this angular momentum loss or redistribution is also uncertain, but an upper limit of $10^{6}$ years has been claimed from mapping the central density at ignition to the expected nucleosynthesis (Yoon \& Langer 2005). This is consistent with our constraints.

\subsection{The companions in SNR 0509-67.5}

In SNR 0509-67.5, Schaefer \& Pagnotta (2012) did not find a candidate for a surviving companion, which they claimed favours the DD scenario. On the other hand, Di Stefano \& Kilic (2012) argued that, if the spin-down timescales is greater than $10^{5} \mathrm{yr}$, the donor star could be too dim to be detectable at the time of explosion. In this paper, we find that the spin-down timescale should usually be shorter than $10^{7} \mathrm{yr}$, but also typically longer than $10^{5} \mathrm{yr}$. If the spin-down timescale is as long as $10^{7} \mathrm{yr}$ while the mass-loss timescale of the ex-companion is shorter then this timescale, e.g. $10^{5} \mathrm{yr}$, the ex-companion in SNR 0509-67.5 could have enough time to cool and to have a luminosity lower than the observational limit (see Fig. 1 in Di Stefano \& Kilic 2012). However, one should keep in mind that the spin-down timescale here has only been constrained for the WD + RG channel. It is unclear whether the spin-down timescale for the WD + MS channel is different from that for the WD + RG channel. In addition, we do not know whether the region around the supernova was empty or contained some CSM, in which case it is unclear whether our calculations are directly relevant to the SNR. Hence, it is too early to arrive at a conclusive conclusion concerning the implica- 


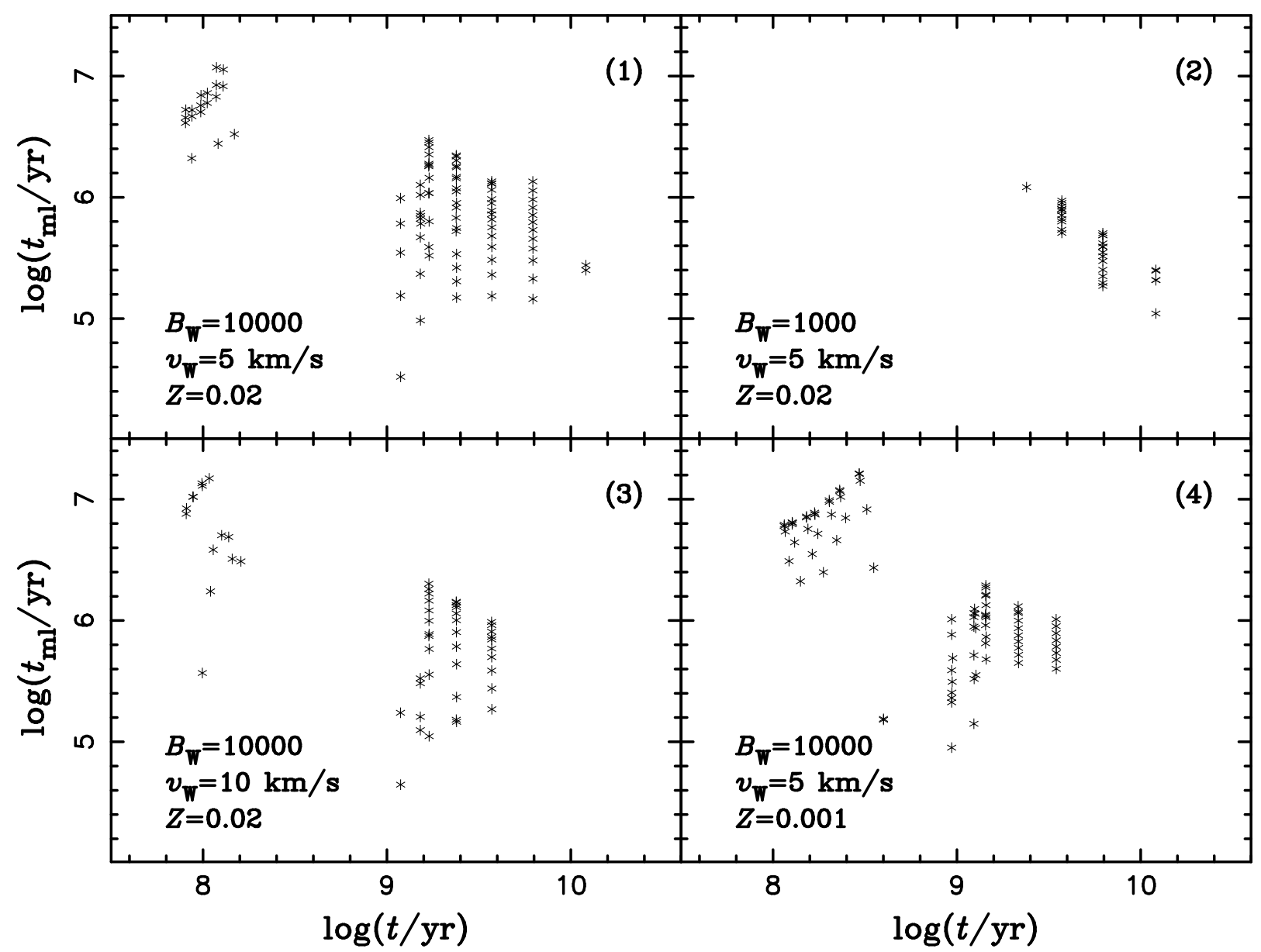

Fig. 4.- Similar to Figure 3 but for different values of $B_{\mathrm{W}}, v_{\mathrm{W}}$ and different metallicities with $M_{W D}^{\mathrm{i}}=1.0 M_{\odot}$, where $Z=0.001$ is the lower metallicity limit allowed in the optically thick wind model (Hachisu et al. 2012). 
tions of the observations of Schaefer \& Pagnotta (2012).

\section{Acknowledgments}

We thank the anonymous referee for his/her constructive suggestions. This work was partly supported by NSFC (11003003,11273012), the Project of Science and Technology from the Ministry of Education (211102) and Key Laboratory for the Structure and Evolution of Celestial Objects, Chinese Academy of Sciences.

\section{REFERENCES}

Alexander, D. R., Ferguson J. W., 1994, ApJ, 437, 879

Boffin, H. M. J. \& Jorissen, A., 1988, A\&A, 205, 155

Chen, X., Tout, C.A., 2007, ChJAA, 7, 2, 245

Chen, X., Han, Z., Tout, C.A., 2011, ApJ, 735, L31

Chen, W., Li, X., 2009, ApJ, 702, 686

Di Stefano, R., Voss, R., Claeys, J. S. W., 2011, ApJ, 738, L1

Di Stefano, R., Kilic, M., 2012, ApJ, 759, 56

Dilday, B., Howell, D.A., Cenko, S.B. et al., 2012, Science, 337, 942

Eggleton, P.P., 1971, MNRAS, 151, 351

González-Hernández J.I., Ruiz-lapuente P., Filippenko A.V., Foley R.J., Gal-Yam A., Simon J.D., 2009, ApJ, 691, 1

Hachisu, I., Kato, M., Nomoto, K., ApJ, 1996, 470, L97

Hachisu, I., Kato, M., Nomoto, K., Umeda, H., 1999a, ApJ, 519, 314

Hachisu, I., Kato, M., Saio, H., Nomoto, K., 2012, ApJ, 744, 69

Han, Z., Podsiadlowski, P., Eggleton, P.P., 1994, MNRAS, 270, 121

Han, Z., Tout, C.A., Eggleton, P.P., 2000, MNRAS, 319, 215
Han, Z., Podsiadlowski, Ph., 2004, MNRAS, 350, 1301

Hillebrandt, W., Niemeyer, J.C., 2000, ARA\&A, 38,191

Howell, D.A. et al., 2009, arXiv: 0903.1086

Iben, I., Tutukov, A.V., 1984, ApJS, 54, 335

Iglesias, C. A., Roger,s F. J., 1996, ApJ, 464, 943

Justham, S., 2011, ApJ, 730, L34

Kato, M., Hachisu I., 2004, ApJ, 613, L129

Kerzendorf W.E., Schmidt B P, Asplund M, et al., 2009, ApJ, 701, 1665

Kerzendorf W.E., Yong D., Schmidt B.P. et al., 2013, 774, 99

Leibundgut, B., 2000, A\&ARv, 10, 179

Leonard D.C., 2007, ApJ, 670, 1275

Maguire, K., Sullivan, M., Patat, F. et al., 2013 , MNRAS.tmp, 2298

Marietta, E., Burrows, A., Fryxell, B., 2000, ApJS, 128, 615

Meng, X., Chen, X., Han, Z., 2007, PASJ, 59, 835

Meng, X., Chen, X., Han, Z., 2008, A\&A, 487, 625

Meng, X., Chen, X., Han, Z., 2009, MNRAS, 395, 2103

Nomoto, K., Thielemann, F-K., Yokoi, K., 1984, ApJ, 286, 644

Patat, F. et al., 2007, Science, 317, 924

Perlmutter, S. et al., 1999, ApJ, 517, 565

Reimers, D., 1975, Mem. R. Soc. liège, 6ième Serie, 8,369

Riess, A. et al., 1998, AJ, 116, 1009

Ruiz-Lapuente, P. et al., 2004, Nature, 431, 1069

Schaefer, B. E., Pagnotta, A., 2012, Nature, 481, 164

Shappee, B.J.; Kochanek, C.S.; Stanek, K.Z., 2013, ApJ, 765, 150 
Sternberg, A., Gal-Yam, A., Simon, J. D. et al., 2011, Science, 333, 856

Tout, C. A., \& Eggleton, P. P. 1988, ApJ, 334, 357

Tout, C. A. \& Hall, D. S., 1991, MNRAS, 253, 9

Wang, B., \& Han, Z., 2012, NewAR, 56, 122

Webbink, R.F., 1984, ApJ, 277, 355

Whelan, J., Iben, I., 1973, ApJ, 186, 1007

Yoon, S.-C., Langer, N., Scheithauer, S., 2004, A\&A, 425, 217

Yoon, S.-C., Langer N., 2004, A\&A, 419, 623

Yoon, S.-C., Langer N., 2005, A\&A, 435, 967

This 2-column preprint was prepared with the AAS LATEX

macros v5.2. 